\title{
Antidepressant Effects and Mechanisms of the Total Iridoids of Valeriana jatamansi on the Brain-Gut Axis
}

Authors

Liwen Wang ${ }^{1 *}$, Yong Sun ${ }^{1}{ }^{*}$, Tingting Zhao ${ }^{1}$, Yongbiao Li ${ }^{1}$, Xiaoqin Zhao ${ }^{1}$, Li Zhang ${ }^{1}$, Lanlan Wu ${ }^{1}$, Liudai Zhang ${ }^{1}$, Tiane Zhang ${ }^{2}$, Guihua Wei ${ }^{1}$, Alvin Altamirano ${ }^{3}$, Yan Tong ${ }^{1 \#}$, Zhiyong Yan ${ }^{1 \#}$

Affiliations

1 School of Life Science and Engineering, Southwest Jiaotong University, Chengdu, PR China

2 School of Basic Medicine, Chengdu University of Traditional Chinese Medicine, Chengdu, PR China

3 Department of Chemistry and Biochemistry, Northern Arizona University, Flagstaff, AZ, USA

Key words

Brain-gut axis, Caprifoliaceae, depression, intestinal flora, iridoids, Valeriana jatamansi

received July 24, 2019

revised November 15, 2019

accepted November 15, 2019

Bibliography

DOI https://doi.org/10.1055/a-1068-9686

Published online December 4, 2019 | Planta Med 2020; 86:

172-179 @ Georg Thieme Verlag KG Stuttgart · New York I

ISSN 0032-0943

Correspondence

Prof. Zhiyong Yan

School of Life Science and Engineering,

Southwest Jiaotong University

No. 111, North 1st Section of Second Ring Road,

Chengdu 610000, China

Phone: + 8618828058165 , Fax: + 8687603202

yzhiy@swjtu.edu.cn

Correspondence

Assistant Professor Yan Tong

School of Life Science and Engineering,

Southwest jiaotong University

No. 111, North 1st Section of Second Ring Road,

Chengdu 610000, China

Phone: + 8613618028642 , Fax: + 8687603202

tongyanmail@126.com

\section{ABSTRACT}

Valeriana jatamansi is widely used in Chinese folk medicine and contains iridoids as important active ingredients. The brain-gut axis describes a complex bidirectional system between the central nervous system and the gastrointestinal tract. Herein, we evaluated the antidepressant effects of total iridoids of Valeriana jatamansi (TIV) and preliminarily investigated the effects of gut microbiota on their antidepressant effects using a chronic, unpredictable mild-stress mouse model. Mice were given $5.7,11.4$, or $22.9 \mathrm{mg} / \mathrm{kg}$ TIV for 1 week. Fluoxetine $(2.6 \mathrm{mg} / \mathrm{kg})$ served as a positive control. Body weight was measured, and behavioral tests including SPT and TST were applied. Colon pathology was assessed through hematoxylin-eosin staining. Additionally, levels of serotonin (5-hydroxytryptamine, 5-HT), norepinephrine (NE), substance $\mathrm{P}$ (SP) and corticotropin-releasing factor (CRF) in the hippocampus and colon were measured by ELISA. In addition, 16SrRNA gene sequencing was performed to explore changes in intestinal microbiota richness and diversity. Our results demonstrated that the model group showed significant depression-like behavior, while the fluoxetine group showed improved depression-like symptoms; after administration, TIV increased body weight, sucrose solution consumption, and ameliorated depression-like behaviors. The overall cell degeneration in colons also improved. In addition, TIV modulated the levels of 5-HT, NE, SP, and CRF expression in the hippocampus and colon. The diversity and richness of gut microbes increased compared to the model group. We therefore conclude that the antidepressant effects of TIV may be related to gut flora structures and regulation of 5-HT, NE, SP, and $\mathrm{CRF}$ in the brain and intestine. 


$\begin{array}{ll}\text { ABBREVIATIONS } \\ \text { 5-HT } & \text { serotonin } \\ \text { CNS } & \text { central nervous system } \\ \text { CRF } & \text { corticotropin-releasing factor } \\ \text { CUMS } & \text { chronic unpredictable mild stress } \\ \text { GF } & \text { germ-free } \\ \text { NE } & \text { norepinephrine } \\ \text { OTUs } & \text { operational taxonomic units } \\ \text { PCoA } & \text { principal coordinate analysis } \\ \text { SP } & \text { substance P } \\ \text { SPF } & \text { specific-pathogen-free } \\ \text { SPT } & \text { test } \\ \text { TIV } & \text { total iridoids rich fraction in V. jatamansi } \\ \text { TIV-H } & \text { high dosage of TIV } \\ \text { TIV-L } & \text { low dosage of TIV } \\ \text { TIV-M } & \text { medium dosage of TIV } \\ \text { TST } & \text { tail suspension test }\end{array}$

\section{Introduction}

Valeriana jatamansi Jones, a plant in the Caprifoliaceae family was initially recorded in the Compendium of Materia Medica [1] and is distributed in many Asian countries [2]. V. jatamansi contains various chemical components such as iridoids, flavonoids, alkaloids, and volatile oils [3]. It has been reported as a psychopharmacological agent and a natural source of valepotriates [4]. Naturally occurring iridoids are among the most important active ingredients of this species as reported by phytochemical investigations. It has been claimed to possess sedative, neuroprotective, anticonvulsant, and antistress activities in various preclinical studies [5]. Antidepressant activities of aqueous, methanol, and aqueous-ethanol extracts of $V$. jatamansi assessed by forced-swim tests and tail suspension tests have also been reported [6]. Furthermore, the antidepressant activity of root essential oil of the $V$. jatamansi patchouli alcohol chemotype has been demonstrated in acute and chronic management studies via forced swim test [7]. Thus, many studies have explored the antidepressant effects of different parts of $V$. jatamansi. Herein we report the antidepressant effect of TIV and explore a potential mechanism of this antidepressant effect.

Depression, also known as depressive disorder, is a chronic mental illness that has been recognized recently by the World Health Organization as the leading cause of disability worldwide [8]. Clinical symptoms of depression include feelings of hopelessness, long-lasting negative moods, cognitive impairment, and suicidal tendencies. This mental disorder, which is highly related to fatigue, productivity loss, and increased mortality, represents an economic burden to public health [9]. Clinically, an estimated $30-60 \%$ of patients treated with existing antidepressants experience little or no relief from depressive symptoms [10].

The brain-gut axis describes a complex bidirectional system between the CNS and the gastrointestinal tract [11]. Gut microbiota in the brain-gut axis profoundly influences brain processes. Recently, gut microbiota has been recognized as a critical component of the brain-gut axis that exerts control at multiple levels rather than only locally in the gastrointestinal tract [12]. Thus, gut micro- biota constitute an ecological community between the brain and the gut that involves multiple overlapping pathways [13].

We therefore established a CUMS mouse model to investigate the effects of TIV on symptoms associated with depression in this model including body weight, tail suspension, and sucrose preference. To evaluate the underlying antidepressant mechanisms of TIV, we also compared the levels of 5-HT, NE, SP, and CRF in the hippocampus and colon, and we evaluated fecal flora. The results of this study may contribute to improving clinical therapeutic effects and further pharmacological studies of $V$. jatamansi.

\section{Results and Discussion}

Depression is a complicated mood disorder, and the occurrence of various stressful events in life is an important cause of depression. Novel candidates for antidepression drugs should be effectively evaluated. In this study, the CUMS mouse model was established to monitor the behavior of mice on the basis of body weight curves, TST, and SPT. The model evaluation results showed that CUMS mice displayed significant depression-like behavior ( $\bullet$ Fig. 1 b, c). Macroscopic characteristics, including the weights of all the mice subjected to CUMS, were lower than those of the mice in the stress-free normal group throughout the study. Compared with those in the CUMS model group without treatment, the mice in the 3 TIV dosage groups demonstrated increased weight during agent administration ( $p<0.01$ or $p<0.05$ ). In addition, the weight increase of the fluoxetine group was the most significant compared with the CUMS group without treatment $(p<0.01$; $>$ Fig. 1 a). After TIV administration, the sucrose preference rate of the fluoxetine group was higher, and the immobile time of the fluoxetine group lower than that of the CUMS model group without treatment $(p<0.01)$; the sucrose preference rate of all treatment groups was increased relative to the CUMS model group without treatment ( $p<0.01$ ); and the immobile time of the model groups without treatment was longer than that of the treatment groups ( $p<0.01$; Fig. 1 c). By monitoring mice behaviors via body weight curve, forced swimming, and TST, TIV demonstrated effective antidepressant activity.

Normal colon tissue structure was observed histopathologically in the stress-free normal group. Compared with the stressfree normal group, the CUMS model group without treatment displayed partial mild vacuolar degeneration, obvious atrophy of the large intestine glands, significant reduction of the number of goblet cells, vacuolar degeneration of a large number of muscle cells, and transparent cytoplasmic lesions. Compared to the CUMS model group without treatment, no vacuoles or obvious pathological changes were visible in the cytoplasm in the fluoxetinetreated CUMS group. In the TIV-treated CUMS groups, overall cell degeneration showed some improvement ( $\triangleright$ Fig.1d). Earlier studies have shown that chronic stress affects the course of inflammatory bowel disease and experimental colitis and may also initiate intestinal inflammation in rats [14]. In pathological conditions, gut microbiota can reach the mesenteric lymph nodes, determining the activation of monocytes and macrophages and the consequent production of inflammatory mediators with antibacterial properties, such as lysozyme [15]. After modeling by CUMS, the depressed mice were accompanied by a certain degree of damage to the colon. After TIV administration, the depression 


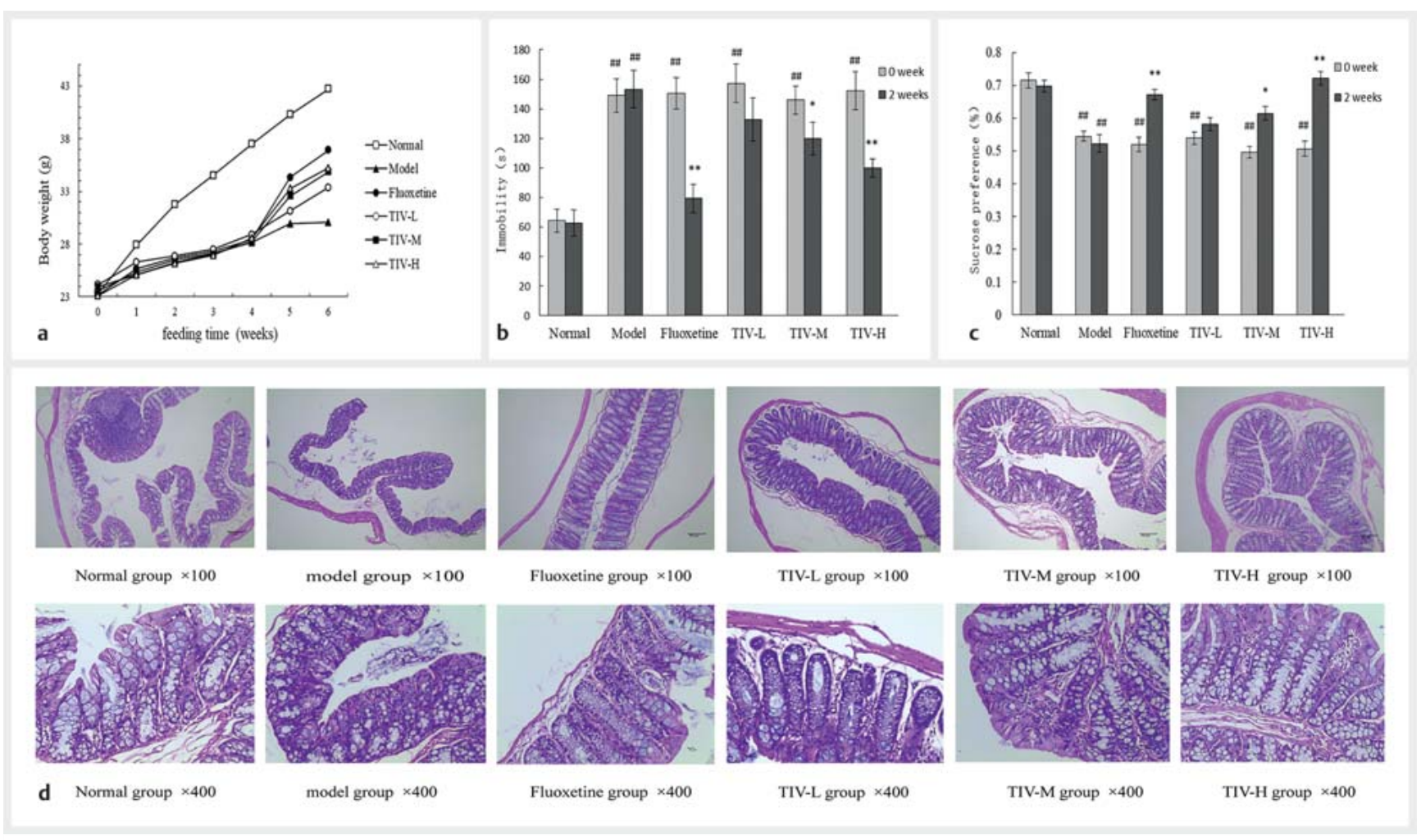

- Fig. 1 a Changes in the body weight of 6 groups. b Sucrose preference in SPT of CUMS mice of TIV. c Immobility time in TST of CUMS mice of TIV. $\mathbf{d}$ Colon histopathological changes were observed by HE staining. Colon pathology changes were observed using a trinocular microscope at $200 \times$ and $400 \times$ total magnifications. Data are reported as the mean \pm SEM. $\# p<0.05$ and $\# \# p<0.01$ versus the normal group. ${ }^{*} p<0.05$ and ${ }^{* *} \mathrm{p}<0.01$ versus the model group.

symptoms and the colon injury improved, suggesting that there is a certain relationship between the brain and the intestine.

The hippocampus in the brain manages human learning, memory, and emotional cognition. A large number of preclinical and clinical studies have shown that depression is associated with the lack of monoamine neurotransmitters in the hippocampus, and regulating monoamine concentrations can ease symptoms of depression [16]. In order to study the effect of TIV on the neurotransmitter between brain and intestine, we mainly studied the brain-gut peptides with double distribution of brain and intestine. Representative brain-gut peptides in the current study included 5-HT, CRF, SP, and NE. Our research found that 5-HT expression in the colons of the model group significantly increased after 2 wk of TIV treatment compared with that of the normal group; by contrast, 5 -HT expression in the hippocampus significantly decreased ( $p<0.01 ;>$ Fig. 2 a). This may be due to a stress-stimulated colonic injury that causes changes in intestinal microbes. Research has shown that microorganisms in the gut can affect the metabolism of tryptophan, which is a precursor of 5-HT. Microorganisms deplete tryptophan through the canine uric acid pathway, thereby decreasing the amount of 5-HT and causing depression symptoms [17]. Compared with that of the CUMS model group without treatment, 5-HT expression in the hippocampus of TIV-M, TIV-H, and fluoxetine groups significantly increased $(p<0.01)$. After administration, TIV may regulate the level of $5-\mathrm{HT}$ by improving the structure of the intestinal flora.
As shown in • Fig. $\mathbf{2}$ b, in the CUMS model group ( $<<0.05)$, NE expression in the hippocampus significantly decreased, whereas that in the colon significantly increased, compared with that in the stress-free normal group. NE expression in the hippocampus of the TIV-H group significantly increased $(p<0.01)$ compared with that of the CUMS model group without treatments.

The expression levels of SP in the hippocampus and colon are shown in - Fig. 2c. After TIV treatment, SP expression significantly increased in the hippocampus and significantly decreased in the colon of the CUMS model group without treatment compared with that in the stress-free normal group $(p<0.01)$. SP expression in the hippocampus of the medium-dose and highdose TIV groups significantly decreased $(p<0.01)$ compared with that in the CUMS model group without treatment. SP expression in the colon of the fluoxetine group significantly decreased $(p<0.01)$. SP plays a corresponding physiological role in the CNS and the digestive system. It can stimulate and accelerate gastrointestinal motility, reduce bile secretion, and possess neurotransmitters and modulation factors [18].

As shown in - Fig. $\mathbf{2 d}$, CRF expression in colon and hippocampus significantly increased in the CUMS model group without treatment $(p<0.01)$, compared with that in the stress-free normal group. Commensal bacteria have been shown to produce neurotransmitters such as dopamine and noradrenaline [19]. In both animal models and humans, CRF is the neurohormone that initiates both the peripheral and central responses to stress and 


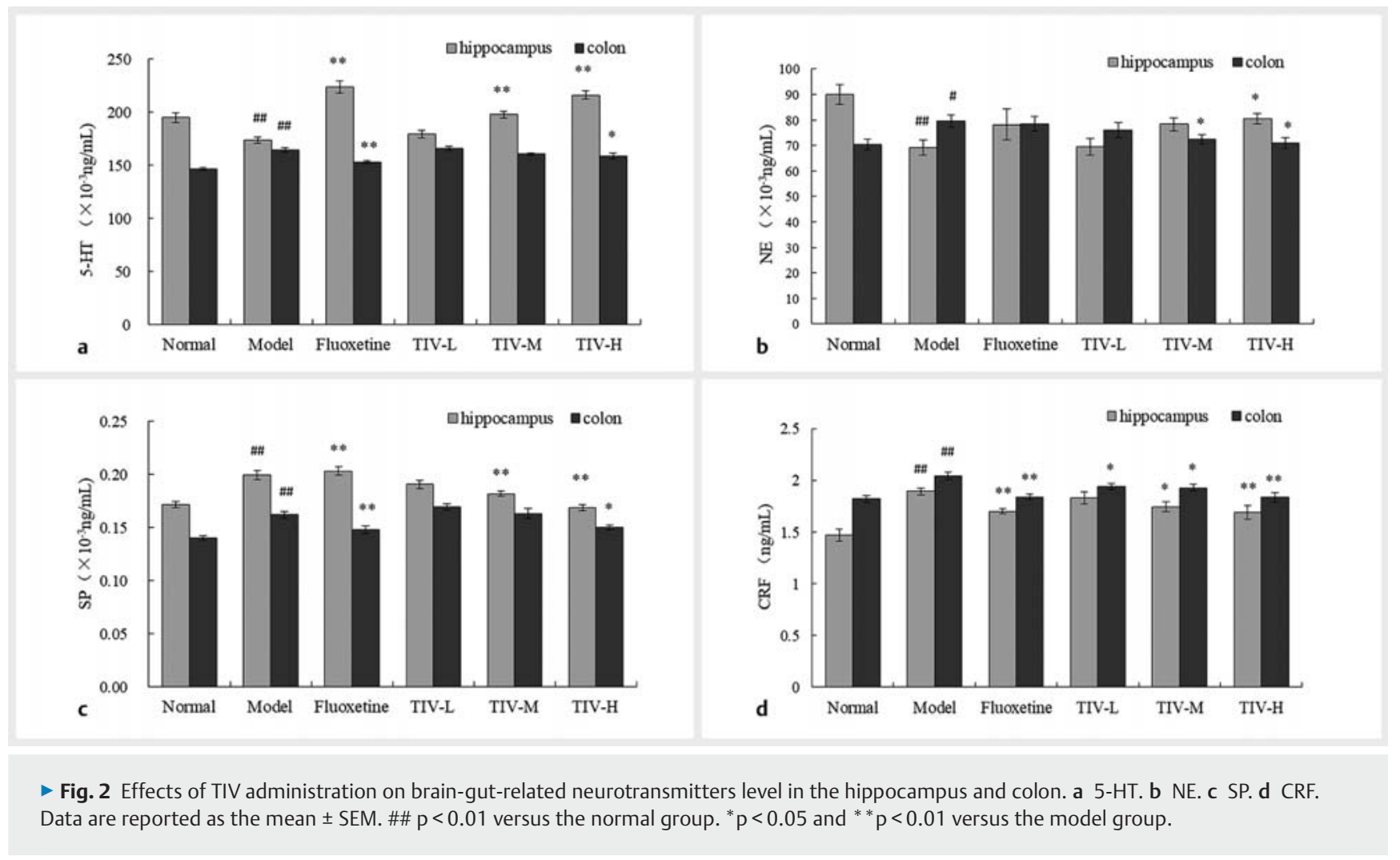

has been shown to be hypersecreted in patients with depression [20]. Compared with that in the CUMS model group without treatment, CRF expression in the hippocampus significantly decreased in the TIV-H and fluoxetine groups $(p<0.01)$. CRF expression in the colon significantly decreased in the TIV-H and fluoxetine groups $(p<0.01)$.

Our study found that the levels of 5-HT, NE, SP, and CRF in the hippocampus and colon of CUMS model mice after TIV treatments were each regulated to some extent. Our results demonstrate that the depressive mouse model established through solitary culture combined with CUMS stress can affect intestinal motility and the release of neurotransmitters and hormones, which may be achieved through the brain-gut axis. In recent y, considerable evidence has shown that intestinal flora plays an important role in regulating mood and brain activities primarily through immunity, the hypothalamus-pituitary-adrenal axis, and neurotransmitters [21]. Microbiota may interact with the nervous system through modulation of host neurotransmitters and/or related pathways. Bacteria have been found to have the capability to produce a range of major neurotransmitters [22-23]. Several studies have shown that intestinal flora participates in the development and maturation of the hypothalamus-pituitary-adrenal axis. Thus, the body's stress response may be affected by directly or indirectly altering the structure of intestinal microorganisms [24].

Gut microbiota communities are relatively stable, contributing to the overall health of the host. Previous studies have confirmed that gut microbiota is inextricably linked to many diseases [25]. Microbiota is also altered in several animal depression models [26]. Sequencing of the 16S rRNA gene was performed to determine the impact of depression on gut microbiota. After TIV treat- ment, the rarefaction curves of all the samples approached the saturation plateau. This result indicates that the current analysis had adequate depth to capture most microbial diversity information ( $\vee$ Fig. 3 a). In our study, PCoA was conducted to compare the similarity in the gut microbiota of 18 specimens. PCoA presented gut microbiota communities in mice from different groups. All TIV groups exhibited certain polymerization tendencies and did not polymerize with the CUMS model group without treatment or the stress-free normal group. The model group was separated from the positive group in PCoA space. This result suggests that the enrichment and diversity of gut microbiota are affected by stress stimuli ( $\bullet$ Fig. $\mathbf{3 b}$ ). Alpha diversity analysis was included for the observed species and the Shannon index, which was intended to be representative of the community richness and community diversity. $>$ Fig. $\mathbf{3} \mathbf{c}$, $\mathbf{d}$ shows the quartile deviation of observed species and the Shannon index. Noticeably, the richness and diversity in mice in the TIV-H group were higher than those of model group by the observed species ( $\vee$ Fig. $3 \mathbf{c}$ ) and the Shannon index ( $\triangleright$ Fig. $\mathbf{3 d}$ ). TIV treatment improved the diversity and richness of mouse gut microbiota caused by depression. At the phylum level, Firmicutes, Proteomicrobiota, and Bacteroidetes were dominant in the mouse gut microbiota samples ( $\bullet$ Fig. 4 b). The most abundant phylum in all the samples was Firmicutes, but the reduction in Firmicutes in the CUMS model group without treatment may be related to the development of depression. A flower diagram was used to show the shared or unique OTUs ( $\vee$ Fig. 4a). The PCoA score plot from sequences at the OTU level with $>97 \%$ similarity indicated that the community composition of the model group (after TIV treatment) was higher than that of the control group. As indicated in $>$ Fig. $\mathbf{4 b}$, all 6 groups shared 


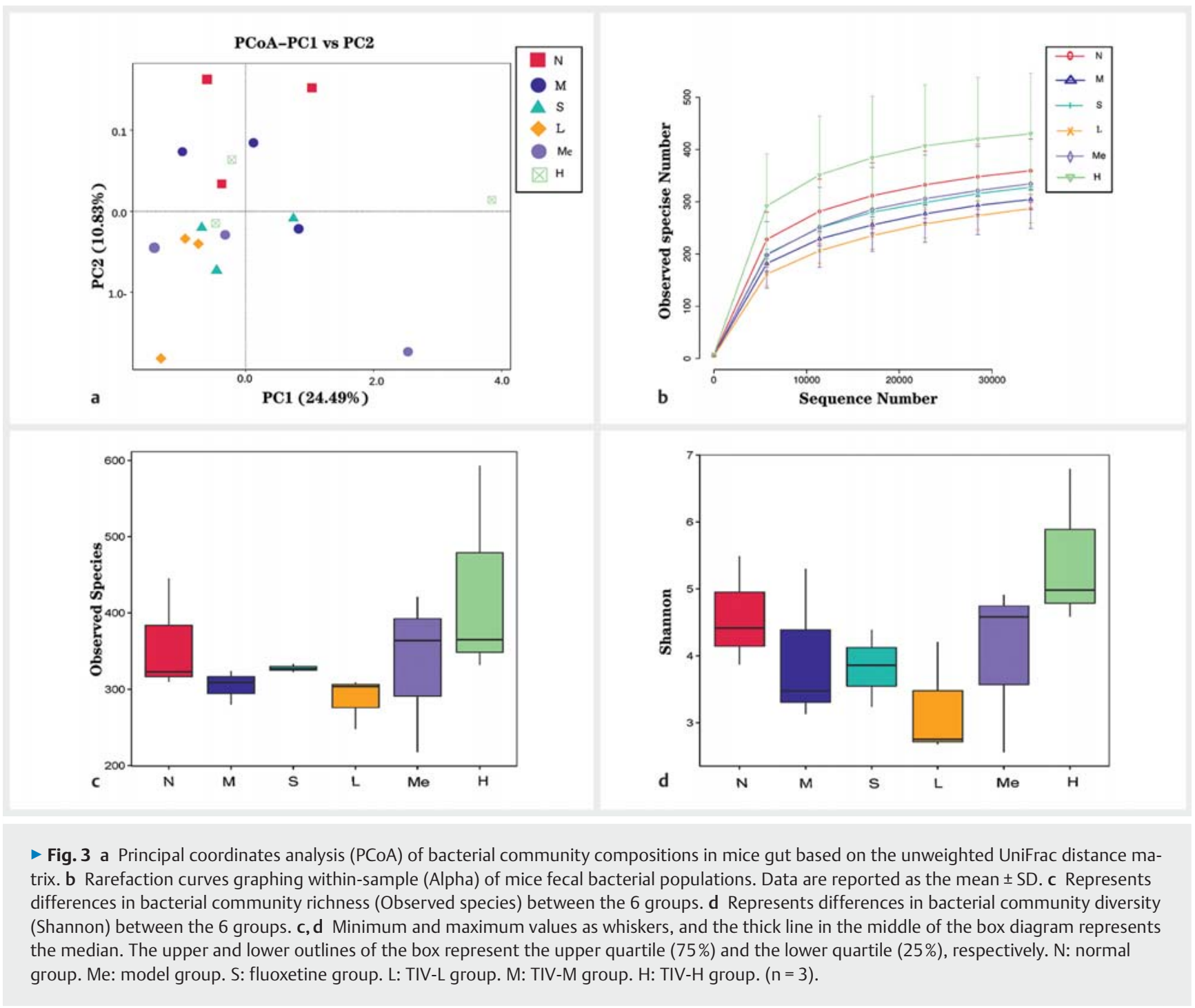

280 OTUs. Unique OTUs were observed in the stress-free normal (29), CUMS model without treatment (2), fluoxetine (3), TIV-L (94), TIV-M (17), and TIV-H (6) groups. It is thus likely that large shared OTUs might be due to the shared similar environment. In this study, we found that the structure of the intestinal flora of mice with depression changed significantly through the sequencing of the 16SrRNA gene. However, our experiments lack a sterile control, sterile animal feeding environments with drinking water, litter, feed, and all items in direct contact with animals being subjected to strict sterilization methods and periodic testing [27]. Studies have found that brain-derived neurotrophic factor, 5-HT, etc. in GF mice have significant changes compared with SPF mice [28], indicating that intestinal microbes significantly affect neurochemistry in the brain. In addition, microbial disorder caused anxiety and social interaction abnormalities. Compared with SPF mice, GF mice showed anxious behavior, and re-suppression of SPF mouse feces facilitated significantly increased anxiety-like behavior in GF mice [29].

Stress has long been known to influence the composition of gut microbiota [30]. Hence, early life stress capable of activating the hypothalamus-pituitary-adrenal axis can affect the development of microbiota and vice-versa, ultimately leading to an imbalance in gut microbiota and an inappropriate stress response [31]. Studies have found the adverse effects of maternal separation upon the microbiota-gut-brain axis in rats in terms of increased colonic visceral sensitivity and disruption to the microbiota themselves. While it was not determined which species of bacteria in the microbiota were affected by maternal separation, this study demonstrated that early life stress has a profound effect upon the gut commensals [32]. Additional evidence of dysregulated microbiota in depression comes from Maes and colleagues [33], who demonstrated that there is bacterial translocation from the gut to the systemic circulation during chronic depression, which would presumably lead to an inflammatory response that may be contributing towards the mood disorder. Many depressed patients have reported less representative microbial diversity, with a relative abundance of Bacteroidetes phylum members and a reduction in Lachnospiraceae family members [34]. Additional clinical evidence has been indirectly obtained by using antimicrobial agents including minocycline to modulate depression [35]. These 


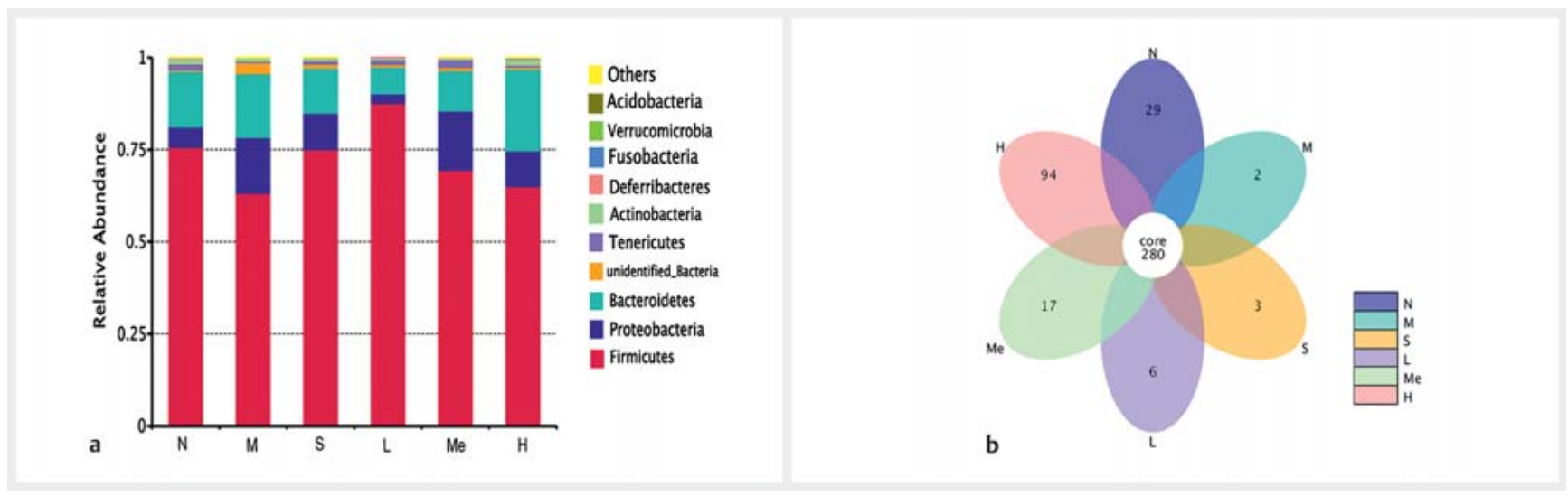

- Fig. 4 a Bacterial community structure variation during decomposition at the phylum level. b Flower diagram showing the unique and shared OTUs in the different groups. N: normal group. M: model group. S: fluoxentine group. L: TIV-L group. M: TIV-M group. H: TIV-H group. ( $n=3$.)

studies have indicated that the stress system and gut microbiota can influence each other in early life. Bacterial commensals in the gut communicate with the CNS via bacterial metabolites, immune mediators, and signaling to the brain directly via the vagus nerve, which regulates brain neurochemistry and behavior [36-37]. Herein we show that TIV improves depression-like symptoms by regulating brain neurotransmitters. The imbalance of intestinal flora structure plays a role in the development of depression, and TIV affects the optimization of the intestinal flora structure. These findings may provide new insights into identifying novel potential etiologies for depression, understanding the role of gut microbiota in psychiatric and CNS disorders, and modulating gut microbiota as a therapeutic tool.

\section{Materials and Methods}

\section{Plant material collection}

The roots and rhizomes of $V$. jatamansi were purchased from Lotus Pond Chinese herbal medicine market in Chengdu, Southwestern China. The samples were identified as V. jatamansi by Professor Liangke Song from the School of Life Science and Engineering, Southwest Jiaotong University. The sample (No. 20181003) is kept in the School of Life Science and Engineering, Southwest jiaotong University, China.

\section{TIV preparation}

TIV was prepared following the previous extraction and purification processes of our research group [38]. Dried powder of V. jatamansi $(10.4 \mathrm{~kg})$ was macerated thrice with $70 \%$ ethanol ( $24 \mathrm{~h}$ each) at room temperature. The solution was then filtered and concentrated under reduced pressure distillation to yield the ethanol extract $(2.32 \mathrm{~kg})$. The extract was diluted with distilled water and underwent ethanol water gradient elution using a D101 macroporous resin column. Then, 95\% ethanol eluent was collected, concentrated into a thick cream, and designated as $\operatorname{TIV}(0.19 \mathrm{~kg} ; 76.5 \%$ purity).

\section{Experimental animals}

A total of 48 healthy SPF adult male Kun Ming (KM) mice ( 6 wk old; weight: 16-20 g), with certificate number SCXK (chuan) 2015030 , were used in this study. All the mice were obtained from Dashuo Biological Technology Company in Chengdu. The mice were housed in plastic cages for a 7-day acclimatization period under normal laboratory conditions of $12 \mathrm{~h}$ light/dark cycles prior to the experiments. The temperature was maintained at $25^{\circ} \mathrm{C} \pm 2{ }^{\circ} \mathrm{C}$. All animals were provided with food (Chengdu Dashuo of Experimental Animals Co., Ltd.) and water ad libitum. The experiments were approved by the Animal Ethics Committee of Southwest Jiaotong University (March 19, 2018, No. S20190319002) and were conducted in compliance with the Guidelines for Animal Experimentation of the university.

\section{Animal groups and model establishment}

After 1 week of acclimatization, $8 \mathrm{KM}$ male mice were randomly selected for the stress-free normal group, and the remaining $40 \mathrm{KM}$ male mice were used to establish the CUMS mouse model, a well-known and effective model to mimic the pathogenesis of depression. All animal groups except for the stress-free normal group were exposed to CUMS. Subsequently, the CUMS groups were housed separately, and stressors were applied for 28 days. The CUMS procedure was performed as indicated previously [39], with a slight modification. In the CUMS procedure, animals are exposed to chronic and continuous low-grade stressors, similar to those associated with human depression. These include the sequential application of various mild stressors: $24 \mathrm{~h}$ food deprivation, $24 \mathrm{~h}$ exposure to a wet cage, $2 \mathrm{~h}$ restricted movement, $5 \mathrm{~min}$ forced swimming in $6-8^{\circ} \mathrm{C}$ water, and tail clamping for $1 \mathrm{~min}$. The mice were subjected to stressors (in random order) for 1 day, without repeating the stressors for 2 consecutive days. The animals in the stress-free normal group were housed in a cage.

\section{Dosing regimens}

To analyze the effects of TIV on mice, the 40 CUMS mice were randomly divided into 5 groups (8 mice per group): CUMS model group without treatment; CUMS model groups treated with lowdose, medium-dose, or high-dose TIV $(5.7,11.4$, and $22.9 \mathrm{mg} / \mathrm{kg} /$ 
$\mathrm{d}$, respectively); and CUMS model group treated with fluoxetine (fluoxetine hydrochloride, $2.6 \mathrm{mg} / \mathrm{kg} / \mathrm{d}$; SuZhou Lilai Pharmaceuticals Co., Ltd.). All agents were administered at a volume of $10 \mathrm{~mL} / \mathrm{kg}$ by the intragastric route once per day for 2 successive wk. The untreated groups (stress-free normal and CUMS model without treatment) received $5 \%$ carboxymethyl cellulose sodium water solution (carboxymethyl cellulose sodium, Chengdu Kelong Chemical Reagent Factory).

\section{Behavior tests}

The TST reflects behavioral despair (hopeless behavior) as observed in patients with depression. The TST was conducted as described [40]. Briefly, in a relatively quiet environment, mice were suspended individually using a suspended tail instrument (ChengDu Taimeng Software Co., Ltd.) for 6 min, and the duration of immobility was recorded during the last 4 min interval of the test. The mice were considered immobile when they hung passively and completely motionless. The test sessions were recorded with a video camera.

The SPT is generally used as an effective means to assess the lack of pleasure in animals. SPT was conducted as described previously [41]. Before the test, the mice were trained to adapt to a sucrose solution $(1 \% \mathrm{w} / \mathrm{v})$. Two bottles of sucrose solution were placed in each cage for 1 day, and 1 bottle of sucrose solution was replaced with water for 1 day. The positions of the 2 bottles were placed randomly and exchanged every $4 \mathrm{~h}$ to avoid the influence of bottle position. Water deprivation (12 h) was implemented before the third day. One bottle of sucrose solution and 1 bottle of water were given $12 \mathrm{~h}$ after the third day. The consumption of sucrose and tap water was recorded after $12 \mathrm{~h}$, and the sucrose consumption rate was calculated as follows: sucrose preference $(\%)=$ sucrose consumption/(water consumption + sucrose consumption) $\times 100 \%$.

\section{Hematoxylin and eosin staining and ELISA}

The mice were sacrificed after the behavioral tests, and hippocampal and colon tissues were collected from each animal. Subsequently, one-third of the colon was fixed with $4 \%$ formaldehyde to observe its pathological changes through hematoxylin and eosin staining. Supernatants of the remaining hippocampal and colon tissues were centrifuged $\left(633 \mathrm{~g}, 15 \mathrm{~min}, 4^{\circ} \mathrm{C}\right)$. The concentrations of 5-HT (ID20181128), NE (ID20181129), SP (ID20181127), and CRF (ID20181128) in the hippocampus and colon were determined using an ELISA kit following the manufacturer's instructions (Jianchen Bioengineering Institute). Concentrations were calculated by referring to a standard curve provided by the manufacturer.

\section{Fecal sample preparation}

The abdominal cavities of the mice were opened through aseptic operation. Colon tissue was cut, and 2 to 3 capsules of feces were extracted and stored in a sterilized cryotube $\left(-80^{\circ} \mathrm{C}\right.$ freezer storage, spare). For each group, 3 cases were randomly sampled as research objects.

\section{DNA extraction and PCR amplification}

The DNA of mouse fecal samples was first extracted using cetyltrimethylammonium bromide. The purity and concentration of DNA were measured via agarose gel electrophoresis, and each sample was diluted to $1 \mathrm{ng} / \mu \mathrm{L}$ with sterile water. The diluted gene was used as a template, the sequencing region was selected, and a specific primer (16S V4 region primers, 515F and 806R) with barcode and amplification was used with related enzymes. PCR amplification was determined through agarose gel electrophoresis, and samples were mixed in equal amounts according to the concentration of each PCR product. Each PCR product was then purified through agarose gel electrophoresis, and the target band was recovered by shearing.

\section{Library construction and computer sequencing}

The V4 region of the 16S rRNA gene was analyzed by high throughput sequencing using the Illumina HiSeq platform by Novogene. Sequences were analyzed using Quantitative Insights into Microbial Ecology software and the UPARSE pipeline. The library was constructed using a library kit; after qubit quantification and library detection, the library was sequenced with Ion S5TMXL (Thermo Fisher).

\section{Statistical analysis}

Experimental data were analyzed using SPSS 16.0. The data were expressed as mean \pm standard error of the mean. Multiple samples were compared through 1-way ANOVA, and the least significant difference test was used to compare 2 groups; $p<0.05$ was considered statistically significant. PCoA was conducted with R3.1.0.

\section{Author Contributions}

Data collection: L. W. Wang, Y. Sun, T. T. Zhao, Y. B. Li, X. Q. Zhao, and Z. Y. Yan; design of the study: L.W. Wang, Y. Sun, T. T. Zhao, Y. B.; statistical analysis: X. Q. Zhao; analysis and interpretation of the data: L. W. Wang, Y. Sun, T.T. Zhao; drafting the manuscript: L.W. Wang, Y. Sun, T. T. Zhao, and Y. B. Li; critical revision of the manuscript: L.W. Wang, Y. Sun, T.T. Zhao, Y.B. Li, X.Q. Zhao, L. Zhang, L. L. Wu, L. D. Zhang, Y. Tong, G. H. Wei, A. Altamirano, T.E. Zhang and Z. Y. Yan.

\section{Acknowledgements}

This research was supported by the Sichuan Province Academic and Technical Leaders Cultivate Support Funds, the Key Project of Research and Development Plan of Science and Technology Department of Sichuan Province (grant number 2018SZ0078, 2018ZR0368), research funds of the Chengdu Science and Technology Bureau (grant number 2015-HM01-00347-SF), and research funds of the Sichuan Traditional Chinese Medicine Administration (grant number 2016Q040).

Conflict of Interest

The authors declare that they have no conflict of interest.

References

[1] Li SZ. Compendium of Materia Medica, 1st ed. China Radio International Press: Beijing, China; 1994: 1639-1643

[2] Schwabe C. Chemistry and biodiversity. Chem Biodivers 2010; 1: 15841587 
[3] Pei QY, Xuan LI, Zhu JX, Chao-Ying MA. Advances in study on pharmacological action and mechanism of Valeriana wallichii DC. Chinese Arch Tradit Chinese Med 2010; 9: 1864-1865

[4] Ernst E. Scientific basis for ayurvedic therapies. Focus Altern Complement Ther 2010; 9: 243

[5] Bhattacharyya D, Jana U, Debnath PK, Sur TK. Initial exploratory observational pharmacology of Valeriana wallichii on stress management: a clinical report. Nepal Med Coll J 2007; 9: 36-39

[6] Fazal S, Nasiara K, Hassan GA, Sewell RDE. Terpenoid content of Valeriana wallichii extracts and antidepressant-like response profiles. Phyther Res 2010; 24: 686-691

[7] Sah SP, Mathela CS, Chopra K. Involvement of nitric oxide (NO) signalling pathway in the antidepressant activity of essential oil of Valeriana wallichii Patchouli alcohol chemotype. Phytomedicine 2011; 18: 1269-1275

[8] Ahmed HU, Hossain MD, Aftab A, Soron TR, Alam MT, Chowdhury MWA, Uddin A. Suicide and depression in the World Health Organization South-East Asia region: a systematic review. WHO South-East Asia J public Heal 2017; 6: 60

[9] Ghanean H, Ceniti AK, Kennedy SH. Fatigue in patients with major depressive disorder: prevalence, burden and pharmacological approaches to management. CNS Drugs 2018; 32: 65-74

[10] Srikumar BN, Paschapur M, Kalidindi N, Adepu B, Das ML, Sreedhara MV, Kuchibhotla VK, PieschI RL, Li YW, Dsp E. Characterization of the adrenocorticotrophic hormone-induced mouse model of resistance to antidepressant drug treatment. Pharmacol Biochem Behav 2017; 161: 53

[11] Cryan JF, O'Mahony SM. The microbiome-gut-brain axis: from bowel to behavior. Neurogastroenterol Motil 2011; 23: 187-192

[12] Collins SM, Michael S, PremysI B. The interplay between the intestinal microbiota and the brain. Nat Rev Microbiol 2012; 10: 735

[13] Quigley EMM. Microbiota-brain-gut axis and neurodegenerative diseases. Curr Neurol Neurosci Rep 2017; 17: 94

[14] Keita AV, Ericson AC, Braaf Y, Wallon C, Söderholm JD. Increased antigen and bacterial uptake in follicle associated epithelium induced by chronic psychological stress in rats. Gut 2004; 53: 494-500

[15] Wang GH, Dong HY, Dong WG, Wang XP, Luo HS, Yu JP, Wang GH, Dong HY, Dong WG, Wang XP. Protective effect of Radix Acanthopanacis Senticosi capsule on colon of rat depression model. World J Gastroenterol 2005; 11: 1373-1377

[16] Marathe SV, D’Almeida PL, Virmani G, Bathini P, Alberi L. Effects of monoamines and antidepressants on astrocyte physiology: implications for monoamine hypothesis of depression. J Exp Neurosci 2018; 12: 117906951878914

[17] Gardier AM. Antidepressant activity: contribution of brain microdialysis in knock-out mice to the understanding of BDNF/5-HT transporter/5-HT autoreceptor interactions. Front Pharmacol 2013; 4: 98

[18] Bravo JA, Dinan TG, Cryan JF. Alterations in the central CRF system of 2 different rat models of comorbid depression and functional gastrointestinal disorders. Int J Neuropsychopharmacol 2011; 14: 666-683

[19] Oleskin AV, El'-Registan GI, Shenderov BA. Role of neuromediators in the functioning of the human microbiota: "business talks" among microorganisms and the microbiota-host dialogue. Mikrobiologiia 2016; 85: $3-25$

[20] Nemeroff CB, Widerlöv E, Bissette G, Walléus H, Karlsson I, Eklund K, Kilts CD, Loosen PT, Vale W. Elevated concentrations of CSF corticotropin-releasing factor-like immunoreactivity in depressed patients. Science 1984; 226: 1342-1344

[21] Rao M, Gershon M. Bugs, guts, and glia: how microbiota influence enteric gliogenesis and migration. Neuron 2015; 85: 229-230

[22] Pokusaeva K, Johnson C, Luk B, Uribe G, Fu Y, Oezguen N, Matsunami RK, Lugo M, Major A, Mori-Akiyama Y. GABA-producing bifidobacterium dentiummodulates visceral sensitivity in the intestine. Neurogastroenterol Motil 2017; 29
[23] Tsavkelova EA, Botvinko IV, Kudrin VS, Oleskin AV. Detection of neurotransmitter amines in microorganisms with the use of high-performance liquid chromatography. Dokl Biochem 2000; 372: 115-117

[24] Tannock GW, Savage DC. Influences of dietary and environmental stress on microbial populations in the murine gastrointestinal tract. Infect Immun 1974; 9: 591-598

[25] Moreau JL. Validation of an animal model of anhedonia, a major symptom of depression. Lencéphale 1997; 23: 280

[26] O'Mahony SM, Marchesi JR, Scully P, Codling C, Ceolho AM, Quigley EMM, Cryan JF, Dinan TG. Early life stress alters behavior, immunity, and microbiota in rats: implications for irritable bowel syndrome and psychiatric illnesses. Biol Psychiatry 2009; 65: 263-267

[27] Gootenberg DB, Turnbaugh PJ. Companion animals symposium: humanized animal models of the microbiome. J Anim Sci 2011; 89: 1531-1537

[28] Gareau MG, Wine E, Rodrigues DM, Cho JH, Whary MT, Philpott D], Macqueen G, Sherman PM. Bacterial infection causes stress-induced memory dysfunction in mice. Gut 2011; 60: 307-317

[29] Desbonnet L, Clarke G, Shanahan F, Dinan TG, Cryan JF. Microbiota is essential for social development in the mouse. Mol Psychiatry 2014; 19 ; $146-148$

[30] Naseribafrouei A, Hestad K, Avershina E, Sekelja M, Linløkken A, Wilson R, Rudi K. Correlation between the human fecal microbiota and depression. Neurogastroenterol Motil Off J Eur Gastrointest Motil Soc 2014; 26 : 1155-1162

[31] Clarke G, Stilling RM, Kennedy PJ, Stanton C, Cryan JF, Dinan TG. Minireview: gut microbiota: the neglected endocrine organ. Mol Endocrinol 2014; 28: 1221-1238

[32] O’Mahony SM, Hyland NP, Dinan TG, Cryan JF. Maternal separation as a model of brain-gut axis dysfunction. Psychopharmacology (Berl) 2011; 214: 71-88

[33] Maes M, Kubera M, Leunis JC, Berk M. Increased IgA and IgM responses against gut ommensals in chronic depression: further evidence for increased bacterial translocation or leaky gut. J Affect Disord 2012; 141: 55-62

[34] Bueno L, de Ponti F, Fried M, Kullak-Ublick GA, Kwiatek MA, Pohl D, Quigley EMM, Tack J, Talley NJ. Serotonergic and non-serotonergic targets in the pharmacotherapy of visceral hypersensitivity. Neurogastroenterol Motil 2010; 19: 89-119

[35] Park AJ, Collins J, Blennerhassett PA, Ghia JE, Verdu EF, Bercik P, Collins SM. Altered colonic function and microbiota profile in a mouse model of chronic depression. Neurogastroenterol Motil 2013; 25: 733 e575

[36] Dinan TG, Stilling RM, Stanton C, Cryan JF. Collective unconscious: how gut microbes shape human behavior. J Psychiatr Res 2015; 63: 1-9

[37] Sherwin E, Rea K, Dinan TG, Cryan JF. A gut (microbiome) feeling about the brain. Curr Opin Gastroenterol 2016; 32: 96-102

[38] Li SH. Studies on Separation and Antianxiety Activity of Iridoids effective Fraction of Valeriana Jatamansi Jones [M.S. Thesis]. Chengdu China: Southwest Jiaotong University; 2013

[39] Biala G, Pekala K, Boguszewska-Czubara A, Michalak A, Kruk-Slomka M, Budzynska B. Behavioral and biochemical interaction between nicotine and chronic unpredictable mild stress in mice. Mol Neurobiol 2017; 54: 904-921

[40] Zhang H, Zhou Z, Chen Z, Zhong Z, Li Z. Ginsenoside Rg3 exerts anti-depressive effect on an NMDA-treated cell model and a chronic mild stress animal model. J Pharmacol Sci 2017; 134: 45-54

[41] Sun J, Wang F, Hong G, Pang M, Xu H, Li H, Tian F, Fang R, Yao Y, Liu J. Antidepressant-like effects of sodium butyrate and its possible mechanisms of action in mice exposed to chronic unpredictable mild stress. Neurosci Lett 2016; 618: 159-166 